\title{
Gallbladder polyp treated with endoscopic polypectomy through a cholecystogastrostomy
}

A 59-year-old man with a history of renal stones presented with the symptoms heartburn over several months. He stated that he had received an abdominal operation for cholecystitis 30 years ago. A diverticulum approximately $7 \mathrm{~cm}$ in diameter with a narrow orifice ( $\bullet$ Fig. 1) was found at the greater curvature of the antrum during upper gastrointestinal endoscopy, with a polypoid mass lesion $(3 \times 3 \mathrm{~cm})$ $(\checkmark$ Fig. 2) in the diverticulum.

Abdominal computed tomography showed the gastric diverticulum (arrow) and a large stone (star) impacted in the distal common bile duct (CBD) without intrahepatic bile duct dilatation ( Fig. 3). The stone in the CBD was removed by endoscopic retrograde cholangiopancreatography (ERCP). The patient received two partial polypectomies of the divertivular polyp; the histology revealed a hyperplastic polyp. At the second endoscopy, we saw a small hole in the diverticulum. We injected contrast medium into the hole under fluoroscopy. The contrast medium went backwards from the cystic duct (arrow) to the CBD and showed the tip of the endoscope in the gallbladder ( $\bullet$ Fig. 4).

Cholecystogastrostomy was performed and explained the impaction of the huge CBD stone without biliary dilatation. Endoscopy through the cholecystogastrostomic orifice showed no residual polyp 6 months later.

Polypoid lesions of the gallbladder can be divided into benign and malignant lesions. Benign polypoid lesions are divided into tumors or pseudotumors. Pseudotumors include polyps, hyperplasia, or other inflammatory lesions. The incidence of benign tumors of resected gallbladders ranges from $0.15 \%$ to $8.5 \%$ [1]. Our patient had a hyperplastic polyp. Laparoscopic cholecystrectomy is advised for polyps larger than $10 \mathrm{~mm}$ or when there is rapid growth. To our knowledge, this is first report of a gallbladder polyp treated with endoscopic polypectomy through cholecystogastrostomy.

Cholecystoenterostomy is a rare complication in gallstone disease, found in 3\%-5\% of patient with cholelithiasis. It often involves the duodenum (71\%), the stomach (14\%), and the colon (6\%). The cholecystogastrostomy in our case was iatrogenic.

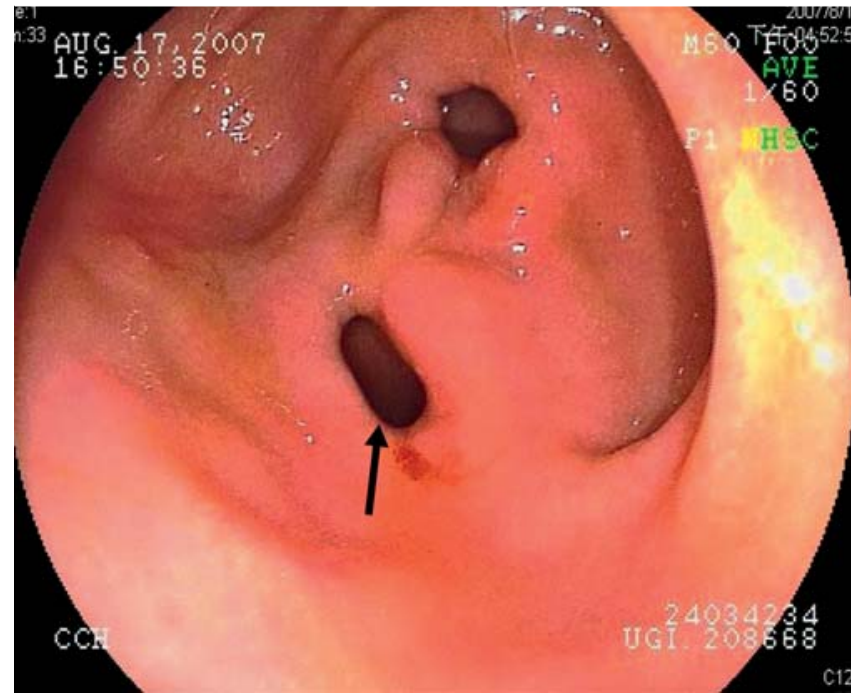

Fig. 1 Diverticulum with a narrow orifice (arrow).

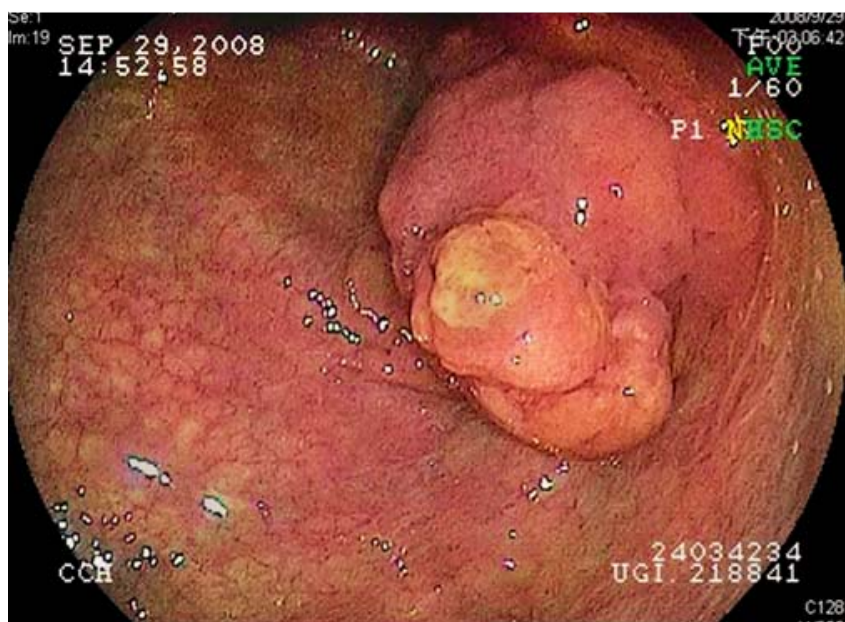

Fig. 2 Polypoid mass lesion in the diverticulum.

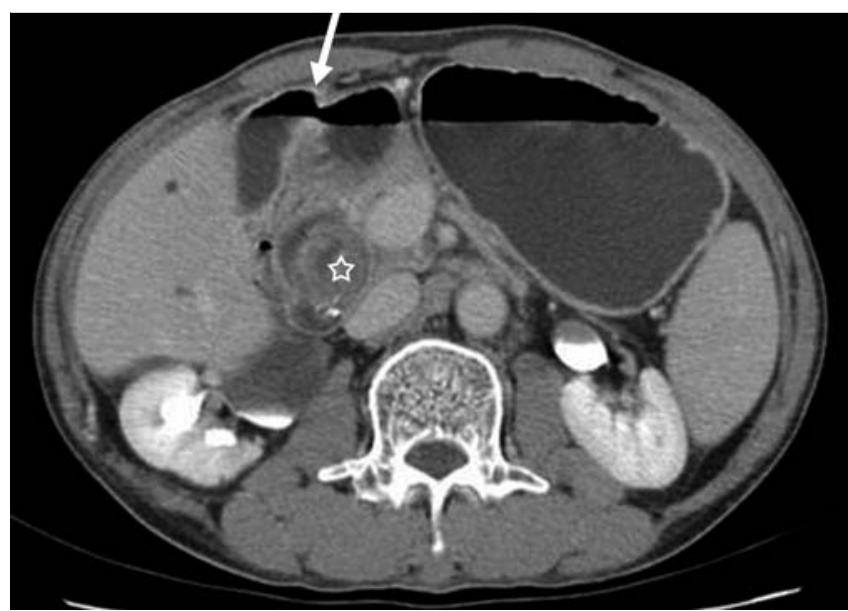

Fig. 3 Abdominal computed tomography showing the gastric diverticulum (arrow) and a large stone (star) impacted in the distal common bile duct (CBD) without intrahepatic bile duct dilatation. 


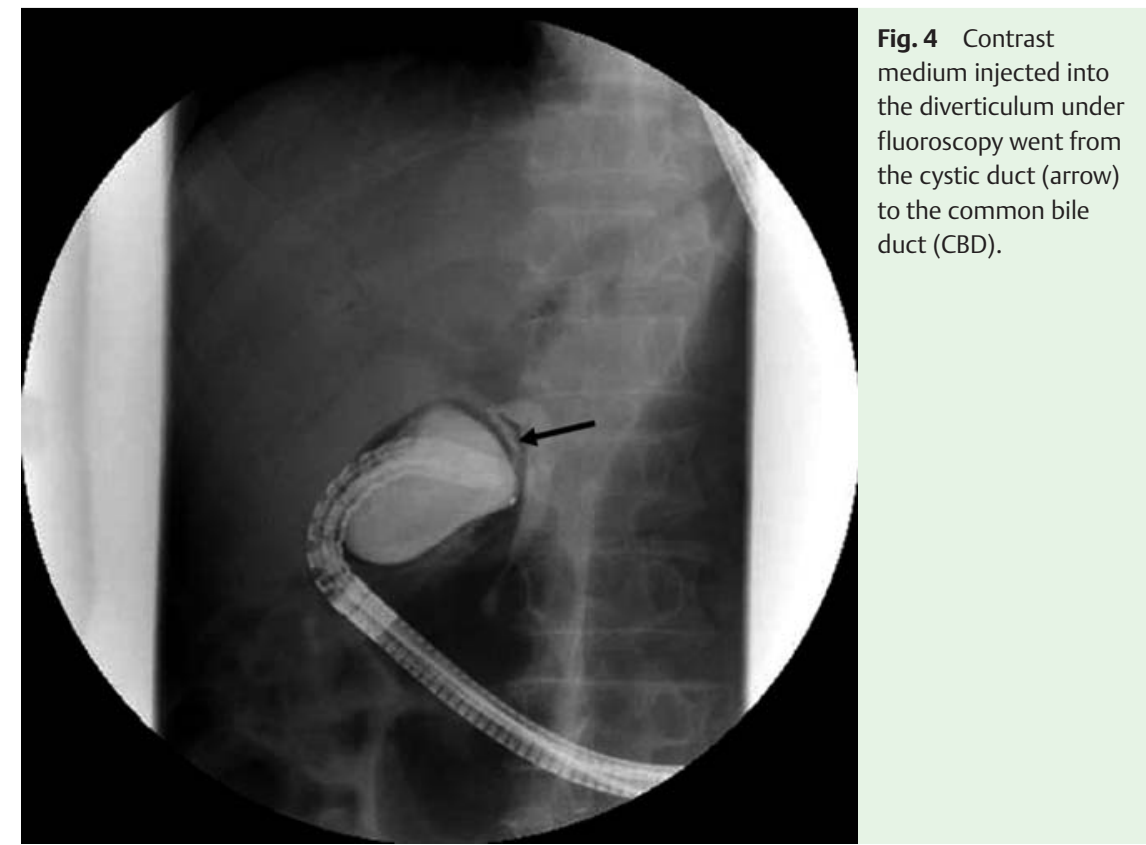

Gastric diverticula are usually asymptomatic, with a prevalence of $0.01 \%$ in autopsy and $0.3 \%$ in radiologic study. Diverticula can be divided into two groups: true (congenital) and pseudo (acquired) [2]. True diverticula are usually located on the posterior wall of the stomach below the gastroesophageal junction, and false diverticula are frequently located in

\section{Y.-Y. Chen ${ }^{1}$, P.-Y. Su ${ }^{2}$, W.-C. Shen ${ }^{1}$}

Department of Gastroenterology, China Medical University Hospital, Changhua, Taiwan

2 Department of Gastroenterology, Changhua Christian Medical Center, Changhua, Taiwan

\section{References}

1 Ljubičić N, Zovak M, Doko $M$ et al. Management of gallbladder polyps: An optimal strategy proposed. Acta Clin Croat 2001; 40: $57-60$

2 Cotea E, Vasilescu A, Dimofte G et al. Gastric diverticulum on the greater curvature. Jurnalul de Chirurgie, Iasi 2007; 3: 269-273

3 LaJoie A, Strum WB. Gastric diverticulum presenting as acute hemorrhage. Gastrointest Endosc 2008; 67: 175 - 176

Bibliography

DOI $10.1055 / \mathrm{s}-0030-1255983$

Endoscopy 2011; 43: E88 - E89

(c) Georg Thieme Verlag KG Stuttgart . New York . ISSN 0013-726X

the gastric antrum [3]. Because the patient presented without epigastric distress, he is receiving regular follow-up at the outpatients' department.

Endoscopy_UCTN_Code_TTT_1AO_2AN

Competing interests: None

\section{Corresponding author}

Y.-Y. Chen, MD

China Medical University Hospital

135 Nanhsiao Street

Changhua

Taiwan

Fax: +886-4-722-8289

27716@cch.org.tw 